International Journal of Difference Equations (IJDE).

ISSN 0973-6069, Volume 15, Number 1, (2020). 1-10

(C) Research India Publications

https://dx.doi.org/10.37622/IJDE/15.1.2020.1-10

\title{
A Glucose-insulin Model with Two Time Delays
}

\author{
Salem Alzahrani* \\ Faculty of Arts and Science in Almandaq, Al-Baha University, Saudi Arabia.
}

\begin{abstract}
A glucose-insulin system with two delays is investigated. By choosing $\tau_{1}$ and $\tau_{2}$ as bifurcation parameters, we show that the Hopf bifurcations occur when time delay crosses some critical values. By analyzing the associated characteristic transcendental equation, the linear stability of the positive equilibrium is investigated and Hopf bifurcation is demonstrated. Numerical simulations are carried out to illustrate the theoretical results and chaotic behaviors are observed.
\end{abstract}

\section{INTRODUCTION}

The dynamic relationship between insulin and their glucose has long been and will continue to be one of the dominant topics, not only in medcine but also in mathematics due to its universal existence and importance. In [1], Andrea De Gaetano and Ovide Arino introduced a following glucose-insulin model:

$$
\begin{aligned}
& \frac{d G}{d t}=-a_{1} x-a_{2} G I+a_{3}, t \in[0, T], \\
& \frac{d I}{d t}=b_{1} G-b_{2} I, t \in[0, T],
\end{aligned}
$$

where

$G \geq 0$ represents glucose concentration,

$I \geq 0$ represents insulin concentration,

$a_{1}$ is the rate constant which represents insulin-independent glucose disappearance,

\footnotetext{
*Corresponding author: Email: salem.bb@hotmail.com

*The research contained in this report has been supported by the University of Al-Baha, Saudi Arabia.
} 
$a_{2}$ is the rate constant which represents insulin-dependent glucose disappearance, $a_{3}$ is the glucose infusion rate,

$b_{1}$ is the rate constant which represents insulin production due to glucose stimulation, $b_{2}$ is the rate constant which represents insulin degradation.

However, this model has attracted the attention of some authors [2]-[5].

Saber and el [3] proposed and briefly discussed the stability of the following delayed glucose-insulin system:

$$
\begin{aligned}
& \frac{d G}{d t}=-a_{1} G(t)-a_{2} G(t-\tau) I(t-\tau)+a_{3}, t \in[0, T], \\
& \frac{d I}{d t}=b_{1} G(t)-b_{2} I(t), t \in[0, T],
\end{aligned}
$$

where $\tau$ represents the time taken by Pancreas to respond to the feedback of the glucose level. In this paper, we will study the stability, and local Hopf bifurcation for the system (1.1) with two time delays $\tau_{1}$ and $\tau_{2}$. In fact, we consider

$$
\begin{aligned}
& \frac{d G}{d t}=-a_{1} G(t)-a_{2} G\left(t-\tau_{1}\right) I\left(t-\tau_{2}\right)+a_{3}, t \in[0, T], \\
& \frac{d I}{d t}=b_{1} G(t)-b_{2} I(t), t \in[0, T] .
\end{aligned}
$$

The initial conditions for system (1.2) take the from

$$
\begin{gathered}
G(\theta)=\phi(\theta), \theta<0, \\
I(\theta)=\psi(\theta), \theta<0,
\end{gathered}
$$

The remainder of the paper is organized as follows. In Section 2, we investigate the stability of the positive equilibrium and the occurrence of local Hopf bifurcations. Moreover, the direction and stability of local Hopf bifurcation are established. In Section 3, numerical simulations are carried out to illustrate the validity of the main results. Some main conclusions are drawn in Section 4.

\section{STABILITY ANALYSIS AND HOPF BIFURCATION}

In this section, we consider the fractional-order systems (1.2). We shall now investigate the dynamics of the delay system (1.2). The equilibrium point of the system (1.2) is one of which

$$
\begin{gathered}
G(t)=G\left(t-\tau_{1}\right) \text { and } \frac{d G}{d t}=0 \\
I(t)=I\left(t-\tau_{2}\right) \text { and } \frac{d I}{d t}=0 .
\end{gathered}
$$


The interior-equilibrium point $E_{1}=\left(G^{*}, I^{*}\right)=\left(\frac{-a d+\sqrt{(a d)^{2}+4 b e c d}}{2 d c}, \frac{-a d+\sqrt{(a d)^{2}+4 b e c d}}{2 e b}\right)$ exists unconditionally as $G^{*}$ and $I^{*}$ are always positive as all the parameters are considered positive. To linearize the model about the equilibrium point $E_{1}$ of model (1.2), let $u_{1}(t)=G(t)-G^{*}, u_{2}(t)=I(t)-I^{*}$. After removing nonlinear terms, we obtain the linear variational system, by using equilibria conditions as

$$
\begin{aligned}
& D u_{1}(t)=-a_{1} u_{1}(t)-a_{2} I^{*} u_{1}\left(t-\tau_{1}\right)-a_{2} G^{*} u_{2}\left(t-\tau_{2}\right), \\
& D u_{2}(t)=b_{1} u_{1}(t)-b_{2} u_{2}(t) .
\end{aligned}
$$

From the linearized model we obtain the characteristic equation

$$
\lambda^{2}+a \lambda+(b \lambda+c) e^{-\lambda \tau_{1}}+d e^{-\lambda \tau_{2}}+f=0,
$$

where $a=a_{1}+b_{2}, b=a_{2} I^{*}, c=a_{2} b_{2} I^{*}, d=a_{2} b_{1} G^{*}, f=a_{1} b_{2}$.

Case 1. $\tau_{1}=\tau_{2}=0$

Lemma 2.1 The positive equilibrium point $E_{1}$ of system (1.2) with $\tau_{1}=\tau_{2}=0$ is locally asymptotically stable.

Proof. For $\tau_{1}=\tau_{2}=0$, Eq. (2.1) becomes

$$
\lambda^{2}-D_{1} \lambda+D_{2}=0
$$

A set of necessary and sufficient conditions that all roots of (2.2) have a negative real part is given in the following form:

$$
D_{1}<0 \text { and } D_{2}>0 .
$$

Then the equilibrium point $E_{1}$ is locally asymptotically stable when the condition (2.2) holds.

Case 2. $\tau_{1}=\tau_{2}=\tau$

For $\tau_{1}=\tau_{2}=\tau$, Eq. (2.1) becomes

$$
\lambda^{2}+a \lambda+(b \lambda+g) e^{-\lambda \tau}+f=0
$$

where $g=c+d$. If $\lambda=i \omega(\omega>0)$ is a root for the characteristic equation (2.4), then $\omega$ should satisfy the following equations

$$
\begin{aligned}
& \omega b \sin \omega \tau+g \omega \cos \omega \tau=\omega^{2}-f, \\
& \omega b \cos \omega \tau-g \omega \sin \omega \tau=-a \omega,
\end{aligned}
$$


which implies that

$$
\omega^{4}+\left(a^{2}-b^{2}-2 f\right) \omega^{2}+f^{2}-g^{2}=0
$$

Since $f+g>0$, we can conclude that if we have the condition:

$$
f-g<0
$$

then $f<g$, and further Eq. (2.5) has a unique positive root

$$
\omega=\sqrt{\frac{-\left(a^{2}-b^{2}-2 f\right) \pm \sqrt{\left(a^{2}-b^{2}-2 f\right)^{2}-4\left(f^{2}-g^{2}\right)}}{2}} .
$$

and the corresponding critical value of the delay is

$$
\tau_{0_{n}}=\frac{1}{\omega_{0}} \arctan \left(-\frac{\omega_{0}\left(a g-b f+b \omega_{0}^{2}\right)}{a b \omega_{0}^{2}+\left(f-\omega_{0}^{2}\right) g}\right)+\frac{2 n \pi}{\omega_{0}}, \quad n=0, \pm 1, \pm 2, \ldots
$$

Define

$$
\tau_{0}=\frac{1}{\omega_{0}} \arctan \left(-\frac{\omega_{0}\left(a g-b f+b \omega_{0}^{2}\right)}{a b \omega_{0}^{2}+\left(f-\omega_{0}^{2}\right) g}\right),
$$

When $\tau_{0}=\tau$, then Eq. (2.4) has a pair of purely imaginary roots $\pm i \omega_{0}$. Differentiating Eq. (2.4) with respect to $\tau$, we obtain

$$
\left[\frac{d \lambda}{d \tau}\right]^{-1}=\frac{2 \lambda+a}{-\lambda\left(\lambda^{2}+a \lambda+f\right)}+\frac{b}{\lambda(b \lambda+g)}-\frac{\tau}{\lambda}
$$

Thus,

$$
\operatorname{Re}\left[\frac{d \lambda}{d \tau}\right]_{\tau=\tau_{0}}^{-1}=\frac{\sqrt{\left(b^{2}-a^{2}+2 f\right)^{2}-4\left(f^{2}-g^{2}\right)}}{g^{2}+b^{2} \omega_{0}^{2}}>0 .
$$

Based on the analysis above and according to the Hopf bifurcation theorem in [6], we have the following results.

Theorem 1 If condition (2.6) is satisfied, then the equilibrium $E_{1}$ is asymptotically stable for all $\tau<\tau_{0}$ and unstable for $\tau>\tau_{0}$. Furthermore, as $\tau$ increase through $\tau_{0}$, $E_{1}$ bifurcates into small amplitude periodic solutions, where $\tau_{0}=\tau_{0 n}$ as $n=0$.

Case 3. $\tau_{1}=0, \tau_{2}>0$

For $\tau_{1}=0, \tau_{2}>0$, Eq. (2.1) becomes

$$
\lambda^{2}+p \lambda+r+d e^{-\lambda \tau_{2}}=0
$$


where $p=a+b, r=c+f$. If $\lambda=i \omega(\omega>0)$ is a root for the characteristic equation (2.7), then $\omega$ should satisfy the following equations

$$
\begin{aligned}
& d \sin \omega \tau_{2}=p \omega, \\
& d \cos \omega \tau_{2}=\omega^{2}-r,
\end{aligned}
$$

which implies that

$$
\omega^{4}+\left(p^{2}-2 r\right) \omega^{2}+r^{2}-d^{2}=0 .
$$

It is easy to see that if the condition

$$
p^{2}-2 r>0, \quad r^{2}-d^{2}>0
$$

holds, then Eq. (2.8) has no positive roots. Hence, all roots of (2.7) have negative real parts when $\tau_{2} \in[0,+\infty)$ under the conditions (2.3) and (2.9).

If (2.3) and

$$
p^{2}-2 r>0, \quad r^{2}-d^{2}<0
$$

hold, then (2.8) has a unique positive root $\omega_{0}^{2}$. Eq. (2.8) has a unique positive root

$$
\omega=\sqrt{\frac{-\left(p^{2}-2 r\right) \pm \sqrt{\left(p^{2}-2 r\right)^{2}-4\left(r^{2}-d^{2}\right)}}{2}} .
$$

and the corresponding critical value of the delay is

$$
\tau_{2_{n}}=\frac{1}{\omega} \arctan \left(\frac{p \omega}{\omega^{2}-r}\right)+\frac{2 n \pi}{\omega_{0}}, n=0, \pm 1, \pm 2, \ldots
$$

Let $\lambda\left(\tau_{2}\right)=\alpha\left(\tau_{2}\right)+i \omega\left(\tau_{2}\right)$ be a root of (2.7) near $\tau_{2}=\tau_{2_{n}}$ and $\alpha\left(\tau_{2}\right)=0, \omega\left(\tau_{2_{n}}\right)=\omega_{0}$. From functional differential equation theory, for every $\tau_{2_{n}}, n=0,1,2, \ldots$, there exists $\varepsilon>0$ such that $\lambda\left(\tau_{2}\right)$ is continuously differentiable at $\tau_{2}$ for $\left|\tau_{2}-\tau_{2_{n}}\right|<\varepsilon$. Substituting $\lambda\left(\tau_{2}\right)$ into the left-hand side of (2.7) and differentiating with respect to $\tau_{2}$, we have

$$
\left(\frac{d \lambda}{d \tau_{2}}\right)^{-1}=\frac{(2 \lambda+p) e^{-\lambda \tau_{2}}}{2 d \lambda}-\frac{\tau_{2}}{\lambda}
$$

which leads to

$$
\begin{aligned}
{\left[\frac{d\left(\operatorname{Re} \lambda\left(\tau_{2}\right)\right)}{d \tau}\right]_{\tau_{2}=\tau_{2_{n}}}^{-1} } & =\left\{\frac{(2 \lambda+p) e^{\lambda \tau_{2}}}{2 d \lambda}\right\}_{\tau_{2}=\tau_{2_{n}}} \\
& =\frac{p^{2}-2 r+2 \omega^{2}}{2 d^{2}}>0 .
\end{aligned}
$$

Noting that

$$
\operatorname{sign}\left\{\frac{d(\operatorname{Re} \lambda)}{d \tau_{2}}\right\}_{\tau_{2}=\tau_{2_{n}}}=\operatorname{sign}\left\{\operatorname{Re}\left(\frac{d \lambda}{d \tau_{2}}\right)^{-1}\right\}_{\tau_{2}=\tau_{2_{n}}}=1 .
$$


we have

$$
\left.\frac{d(\operatorname{Re} \lambda)}{d \tau_{2}}\right|_{\tau_{2}=\tau_{2_{n}}}>0
$$

According to the above analysis and Corollary 2.4 of [7], we have the following results.

Theorem 2 For $\tau_{1}=0$, assume that (2.3) is satisfied. Then the following conclusions hold:

(i) If (2.9) holds, then the positive equilibrium $E_{1}$ of the system (1.2) is asymptotically stable for all $\tau_{2} \geq 0$.

(ii) If (2.10) holds, then the positive equilibrium $E_{1}$ of the system (1.2) is asymptotically stable for $\tau_{2}<\tau_{2_{0}}$ and unstable for $\tau_{2}>\tau_{2_{0}}$. Furthermore, the system (1.2) undergoes a Hopf bifurcation at the positive equilibrium $E_{1}$ when $\tau_{2}=\tau_{2_{0}}$.

Case 4. $\tau_{2}=0, \tau_{1}>0$

For $\tau_{2}=0, \tau_{1}>0$, Eq. (2.1) becomes

$$
\lambda^{2}+a \lambda+(b \lambda+c) e^{-\lambda \tau_{1}}+f=0
$$

If $\lambda=i \omega(\omega>0)$ is a root for the characteristic equation (2.11), then $\omega$ should satisfy the following equations

$$
\begin{aligned}
& \omega b \sin \omega \tau_{1}+c \omega \cos \omega \tau_{1}=\omega^{2}-f, \\
& \omega b \cos \omega \tau_{1}-c \omega \sin \omega \tau_{1}=-a \omega,
\end{aligned}
$$

which implies that

$$
\omega^{4}+\left(a^{2}-b^{2}-2 f\right) \omega^{2}+f^{2}-c^{2}=0 .
$$

Since $f+c>0$, we can conclude that if we have the condition:

$$
f-c<0,
$$

then $f<c$, and further Eq. (2.12) has a unique positive root

$$
\omega=\sqrt{\frac{-\left(a^{2}-b^{2}-2 f\right) \pm \sqrt{\left(a^{2}-b^{2}-2 f\right)^{2}-4\left(f^{2}-c^{2}\right)}}{2}} .
$$

and the corresponding critical value of the delay is

$$
\tau_{1_{n}}=\frac{1}{\omega_{0}} \arctan \left(-\frac{\omega_{0}\left(a g-b f+b \omega_{0}^{2}\right)}{a b \omega_{0}^{2}+\left(f-\omega_{0}^{2}\right) c}\right)+\frac{2 n \pi}{\omega_{0}}, \quad n=0, \pm 1, \pm 2, \ldots
$$


Define

$$
\tau_{1}=\frac{1}{\omega_{0}} \arctan \left(-\frac{\omega_{0}\left(a c-b f+b \omega_{0}^{2}\right)}{a b \omega_{0}^{2}+\left(f-\omega_{0}^{2}\right) c}\right),
$$

When $\tau_{1_{0}}=\tau_{1}$, then Eq. (2.11) has a pair of purely imaginary roots $\pm i \omega_{0}$. Differentiating Eq. (2.11) with respect to $\tau_{1}$, we obtain

$$
\left[\frac{d \lambda}{d \tau_{1}}\right]^{-1}=\frac{2 \lambda+a}{-\lambda\left(\lambda^{2}+a \lambda+f\right)}+\frac{b}{\lambda(b \lambda+c)}-\frac{\tau_{1}}{\lambda} .
$$

Thus,

$$
\operatorname{Re}\left[\frac{d \lambda}{d \tau}\right]_{\tau_{1}=\tau_{1_{0}}}^{-1}=\frac{\sqrt{\left(b^{2}-a^{2}+2 f\right)^{2}-4\left(f^{2}-c^{2}\right)}}{c^{2}+b^{2} \omega_{0}^{2}}>0 .
$$

Based on the analysis above and according to the Hopf bifurcation theorem in [6], we have the following results.

Theorem 3 If condition (2.13) is satisfied, then the equilibrium $E_{1}$ is asymptotically stable for all $\tau<\tau_{0}$ and unstable for $\tau>\tau_{0}$. Furthermore, as $\tau_{1}$ increase through $\tau_{1_{0}}$, $E_{1}$ bifurcates into small amplitude periodic solutions, where $\tau_{1_{0}}=\tau_{1_{0} n}$ as $n=0$.

\section{NUMERICAL SIMULATIONS}

In this section, we present some examples and numerical simulations to verify our theoretical results proved in the previous sections by using matlab programm.

Example 3.1 We consider the system (2.1) in the case $\tau_{1}=\tau_{2}=0$. By taking $a_{1}=0.01135, a_{2}=1.714, a_{3}=1.223, b_{1}=0.0722, b_{2}=1.3272$. In this case, the numerical simulation (Figure 1) shows that both the glucose and insulin diverges. There is a positive equilibrium $E_{1}=\left(G^{*}, I^{*}\right)=(3.5613,0.1937)$. By Theorem 1 in [2], i.e., the interior-equilibrium point $E_{1}$ is unstable when $\tau_{1}=\tau_{2}=0$ since

$$
\left(b_{1}-a_{2} G^{*}\right)^{2}=36.3836>4 b_{2}\left(a_{1}+a_{2} I^{*}\right)=1.823 .
$$

Example 3.2 We consider the system (2.1) in the case $\tau_{1}=\tau_{2}=\tau, \tau \neq 0$. By taking $a_{1}=0.01135, a_{2}=1.714, a_{3}=1.223, b_{1}=0.0722, b_{2}=1.3272$ with time delay $\tau=2.2362$. There is a positive equilibrium $E^{*}=\left(G^{*}, I^{*}\right)=(3.5613,0.1937)$. It means the system (1) is stable on $E^{*}=(120.2304,2.8182)$. By Theorem 2.2, there is a critical value $\tau_{0}=2.2362$. The computer simulations (Fig.2), (Fig.3), (Fig.4), (Fig.5), show that $E_{1}$ is asymptotically stable when $\tau=2.0362<\tau_{0}=2.2362$. When $\tau$ passes through the critical value $\tau_{0}=2.2362, E_{1}$ loses its stability and a Hopf bifurcation occurs, i.e., a family of periodic solutions bifurcate from $E_{1}$. When $\tau=2.5362>\tau_{0}=2.2362, E_{1}$ is unstable, see (Fig.6), (Fig.7). 


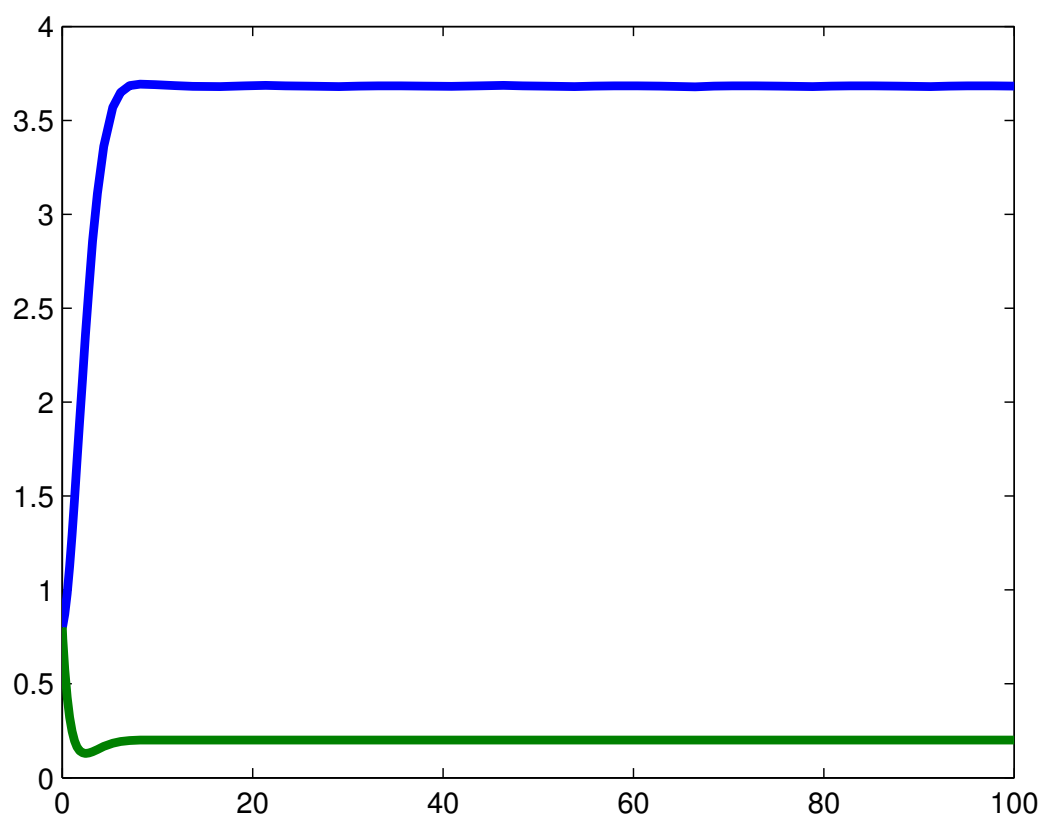

Figure 1: Phase plain of the Glucose-Insulin dynamics for $\tau_{1}=\tau_{2}=0$

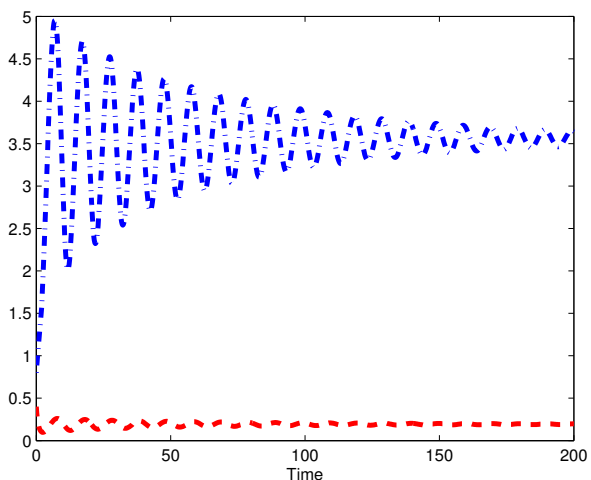

Figure 2: Glucose-Insulin dynamics for $\tau=1.0738$

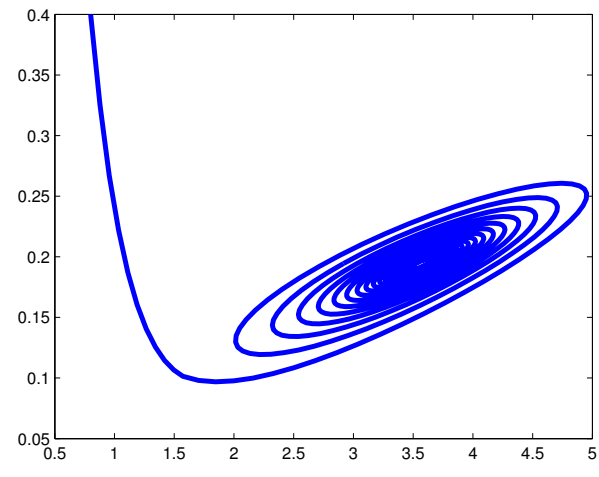

Figure 3: Phase plain of the Glucose-Insulin dynamics for $\tau=1.0738$ 


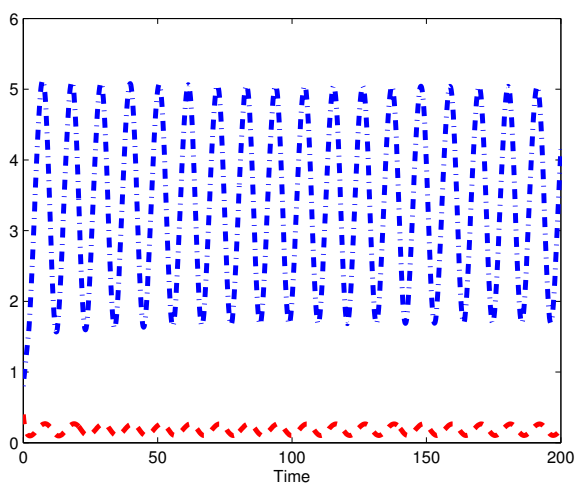

Figure 4: Glucose-Insulin dynamics for $\tau=1.2738$

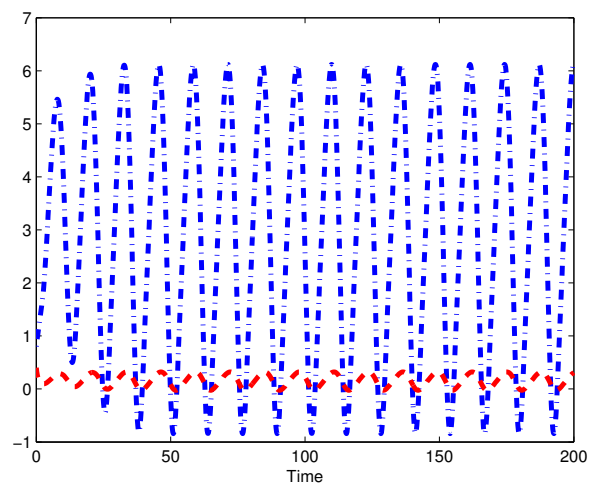

Figure 6: Glucose-Insulin dynamics for $\tau=1.3738$

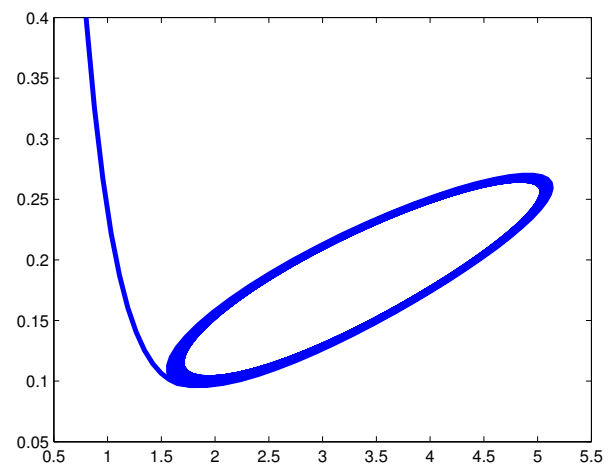

Figure 5: Phase plain of the Glucose-Insulin dynamics for $\tau=1.2738$

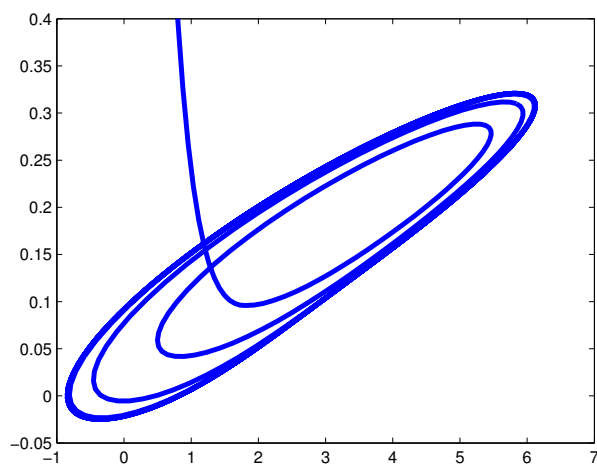

Figure 7: Phase plain of the Glucose-Insulin dynamics for $\tau=1.3738$

\section{CONCLUSION}

Delay differential equations are an interesting form of differential equations, with many different applications, particularly in the biological and medical worlds. In this paper, a mathematical model has been proposed and analyzed to study the dynamics of glucose and insulin in the human body. Numerical simulations are carried out to demonstrate the results obtained. Our results reveal the conditions on the parameters so that the periodic solution exist surrounding the interior equilibrium. Furthermore, the direction of Hopf bifurcation and the stability of bifurcated periodic solutions are investigated. From the above results, we conclude that the model is physiologically consistent and 
may be a useful tool for further research on diabetes.

\section{ACKNOWLEDGMENT}

This research is a part of a project entitled "Different Strategies for Diabetes Diseases in Al-Baha region, Saudi Arabia by Mathematical Modelling”. This project was funded by the Deanship of Scientific Research, Albaha University, KSA (Grant No. 1439/019). The assistance of the deanship is gratefully acknowledged.

\section{REFERENCES}

[1] Andrea De Gaetano, Ovide Arino, Mathematical modelling of the intravenous glucose tolerance test, Journal of Mathematical Biology, February 2000, Volume 40, Issue 2, pp 136-168.

[2] J. Hussain and D. Zadeng, A mathematical model of glucose-insulin interaction, Science Vision, 14 (2), 84-88, 2014.

[3] Saber Sayed, Bashier EBM, Alzahrani SM and Noaman IA, A Mathematical Model of Glucose-Insulin Interaction with Time Delay. J Appl. Computat Math 2018, Vol 7(3): 416.

[4] Saber Sayed, Alzahrani SM, Stability Analysis of a Fractional Order Delayed Glucose-Insulin Model, Albaha University Journal of Basic and Applied Sciences. 2019; 3(2):7-14.

[5] Saber Sayed, Alzahrani SM, Hopf Bifurcation on Fractional Ordered Glucose-Insulin System with Time-Delay, Albaha University Journal of Basic and Applied Sciences 3(2) (2019) 27-34.

[6] Hassard, BD, Kazarinoff, ND, Wan, YH: Theory and Applications of Hopf Bifurcation. Cambridge University Press, Cambridge (1981)

[7] Ruan, S. and Wei, J. On the zero of some transcendential functions with applications to stability of delay differential equations with two delays, Dynamics of Continuous, Discrete and Impulsive Systems Series A 10(1): (2003) 863-874. 